\title{
Filopatría de la ballena jorobada (Megaptera novaeangliae Borowski, 1781), al área de alimentación del estrecho de Magallanes
}

\author{
Site Fidelity of Humpback Whales (Megaptera novaeangliae Borowski, 1781) to the \\ Magellan Strait feeding ground \\ Jorge A. Acevedo ${ }^{1,2}$, Anelio Aguayo-Lobo ${ }^{1,3}$ y Luis A. Pastene ${ }^{4}$ \\ ${ }^{1}$ Fundación Centro de Estudios del Cuaternario (CEQUA). Avda. Bulnes 01890, Punta Arenas, Chile \\ ${ }^{2}$ Magíster (c) Universidad de Magallanes-Universidad de Concepción \\ ${ }^{3}$ Instituto Antártico Chileno, Departamento Científico. Plaza Muñoz Gamero 1055, Punta Arenas, Chile \\ ${ }^{4}$ Institute of Cetacean Research, Toyomi-cho 4-5, Chuo-Ku, Tokio, Japón \\ jacevedo@123mail.cl
}

\begin{abstract}
Resumen.- La ballena jorobada migra entre las áreas de alimentación en las altas latitudes y las áreas de reproducción en las bajas latitudes, exhibiendo un marcado grado de filopatría hacia sus destinos migratorios. La ballena jorobada del Pacífico suroriental migra entre el área de reproducción, principalmente en las aguas de Ecuador y Colombia, y el área de alimentación alrededor de la península Antártica. Estudios previos han sugerido una segunda área de alimentación para esta población, localizada en las aguas del estrecho de Magallanes. El presente estudio evalúa la filopatría de la ballena jorobada esta segunda área de alimentación en el estrecho de Magallanes, usando datos de foto-identificación obtenidos durante 10 cruceros de avistamiento realizados en tres veranos australes consecutivos (2002-2003 a 2004-2005). Si el estrecho de Magallanes constituye una segunda área de alimentación para una parte de los animales de la población del Pacífico suroriental, entonces se debería esperar un alta proporción de retorno de esos animales foto-identificados a esta área, en diferentes veranos australes. La filopatría de los ejemplares foto-identificados a esta segunda área de alimentación en el estrecho de Magallanes muestra ser alto ( $\bar{X}=78,9 \%)$. Además, la filopatría de los ejemplares no mostró ser significativamente diferentes entre sexos ni entre categorías de clase/edad. Finalmente, la alta tasa de retorno de los ejemplares foto-identificados en los diferentes veranos australes, indica que el estrecho de Magallanes constituye otra zona de alimentación para, al menos, una parte de la población de ballena jorobada del Pacífico suroriental.
\end{abstract}

Palabras clave: Cetacea, Pacífico suroriental, fotoidentificación, Chile

\begin{abstract}
Humpback whales migrate annually between high latitude feeding grounds and low latitude breeding grounds, exhibiting a marked degree of fidelity to migratory destinations. Southeastern Pacific humpback whales migrate between the reproductive area located principally off Ecuador and Colombia and the feeding area located around the Antarctic Peninsula. Previous studies suggested a second feeding area for this population in the Magellan Strait. This study evaluates the site fidelity of humpback whales to the Magellan Strait feeding area using photo-identification data obtained during 10 sighting cruises carried out in three austral summer seasons (2002-2003 to 2004-2005). If the Magellan Strait is a second feeding area for some whales of this population, a high return rate of the same animals photoidentified to this area than by chance would be expected. The site fidelity of humpback whales in different austral summer seasons shown a high return rate $(\overline{\mathrm{X}}=78.9 \%)$. Furthermore no significant differences were found in the return rate between female and males or between different class/age categories. Finally, the high return rate humpback whale, indicate that Magellan Strait is another feeding ground at least for a part of the Southeastern Pacific humpback whales population.
\end{abstract}

Key words: Cetacea, Southeast Pacific, photo-identification, Chile 


\section{Introducción}

La ballena jorobada (Megaptera novaeangliae Borowski 1781), al igual que otras especies de la familia Balaenopteridae, realiza migraciones estacionales entre sus áreas de reproducción en bajas latitudes y las áreas de alimentación en altas latitudes (Rice 1998). La excepción son las ballenas jorobada que habitan en el Mar Arábico, las cuales son consideradas residentes (Mikhalev 1997). Varios autores han planteado la hipótesis de que no todos los individuos migrarían a las áreas de reproducción, permaneciendo algunos en las áreas de alimentación durante todo el año (Chittleborough 1958, Brown et al. 1995, Craig \& Herman 1997), aunque la clase de sexo/edad de estos animales aún es desconocida (Simmons \& Marsh 1985, Chaloupka \& Osmond 1999, Clapham 2000).

Diferentes tasas de filopatría ${ }^{1}$ hacia sus respectivas áreas de reproducción y de alimentación han sido sugeridos para las poblaciones de $M$. novaeangliae (Calambokidis et al. 1997², Calambokidis et al. 2001, Waite et al. 1999), sin embargo, existe un consenso, con base en estudios utilizando la técnica de fotoidentificación, que la filopatría de la ballena jorobada a sus áreas de alimentación es más alta que a sus áreas de reproducción (Calambokidis et al. 2001); no obstante, no existe consenso sobre las diferencias de filopatría entre machos y hembras.

Craig \& Herman (1997) sugieren que los machos muestran una mayor filopatría que las hembras en el área de reproducción de Hawaii. En la misma línea, Calambokidis et al. (2000) informan que los machos

\footnotetext{
1 Definida como la tendencia de un animal a permanecer o retornar en años subsecuentes a un lugar específico (Soler et al. 2001). En el caso de las especies migratorias como los Balaenopteridae, este término también es ampliamente usado para las áreas de alimentación.

2 Calambokidis J, G Steiger, JM Straley, TJ Quinn II, LM Herman, S Cerchio, DR Salden, M Yamaguchi, F Sato, J Urbán, J Jacobsen, O von Ziegesar, KC Balcomb, CM Gabriele, ME Dahlheim, N Higashi, S Uchida, JKB Ford, Y Miyamura, P Ladrón de Guevara, SA Mizroch, L Schlender \& $\mathbf{K}$ Rasmussen. 1997. Abundance and population structure of humpback whales in the North Pacific basin. Final Report. Submitted to Southwest Fisheries Science Center, La Jolla, California. 82 pp.
}

foto-identificados en el área de alimentación de California (Pacífico nororiental) tienen una mayor probabilidad de ser observados en el área de reproducción de México que en el caso de las hembras. Por otra parte, Garrigue et al. (2000) concluyen que los machos serían más móviles que las hembras entre las áreas de reproducción de Oceanía oriental.

La población de ballena jorobada del Pacífico suroriental realiza sus migraciones anuales entre su área de reproducción localizada principalmente en las aguas de Ecuador y Colombia y el área de alimentación localizada al sur de la Convergencia Antártica, en la costa occidental de la península Antártica (Omura 1953, Mackintosh 1965, Garrigue et al. 2002, Stevick et al. 2004). Gibbons et al. (2000), basados en datos de avistamientos obtenidos durante el verano austral de 1999, propusieron que las aguas del estrecho de Magallanes (Chile continental) constituiría otra posible área de alimentación para la ballena jorobada del Pacífico suroriental. Posteriormente, Gibbons et al. (2003), basados en datos de foto-identificación, sugirieron cierto nivel de filopatría de los animales a esta posible área de alimentación, pero sus datos fueron insuficientes para obtener resultados concluyentes.

Basado en lo anterior, si las aguas del estrecho de Magallanes constituyen otra área de alimentación para la ballena jorobada del Pacífico suroriental, se debería esperar que los animales foto-identificados en el estrecho de Magallanes presentaran un alta tasa de retorno a estas aguas en los veranos australes. Esta hipótesis es evaluada en este estudio mediante el análisis de datos de foto-identificación obtenidos sistemáticamente durante tres veranos australes consecutivos en el estrecho de Magallanes y canales adyacentes.

\section{Materiales y Método}

\section{Área de Estudio}

El área de estudio abarca las aguas de la porción occidental y central del estrecho de Magallanes hasta Paso Froward, incluyendo el golfo de Xaultegua y canal Abra; así como los canales y bahías exteriores localizadas al sur de las islas Capitán Aracena y Clarence y el SE de la isla Santa Inés, que incluyen los canales Magdalena, Cockburn, Bárbara y González (Fig. 1). 


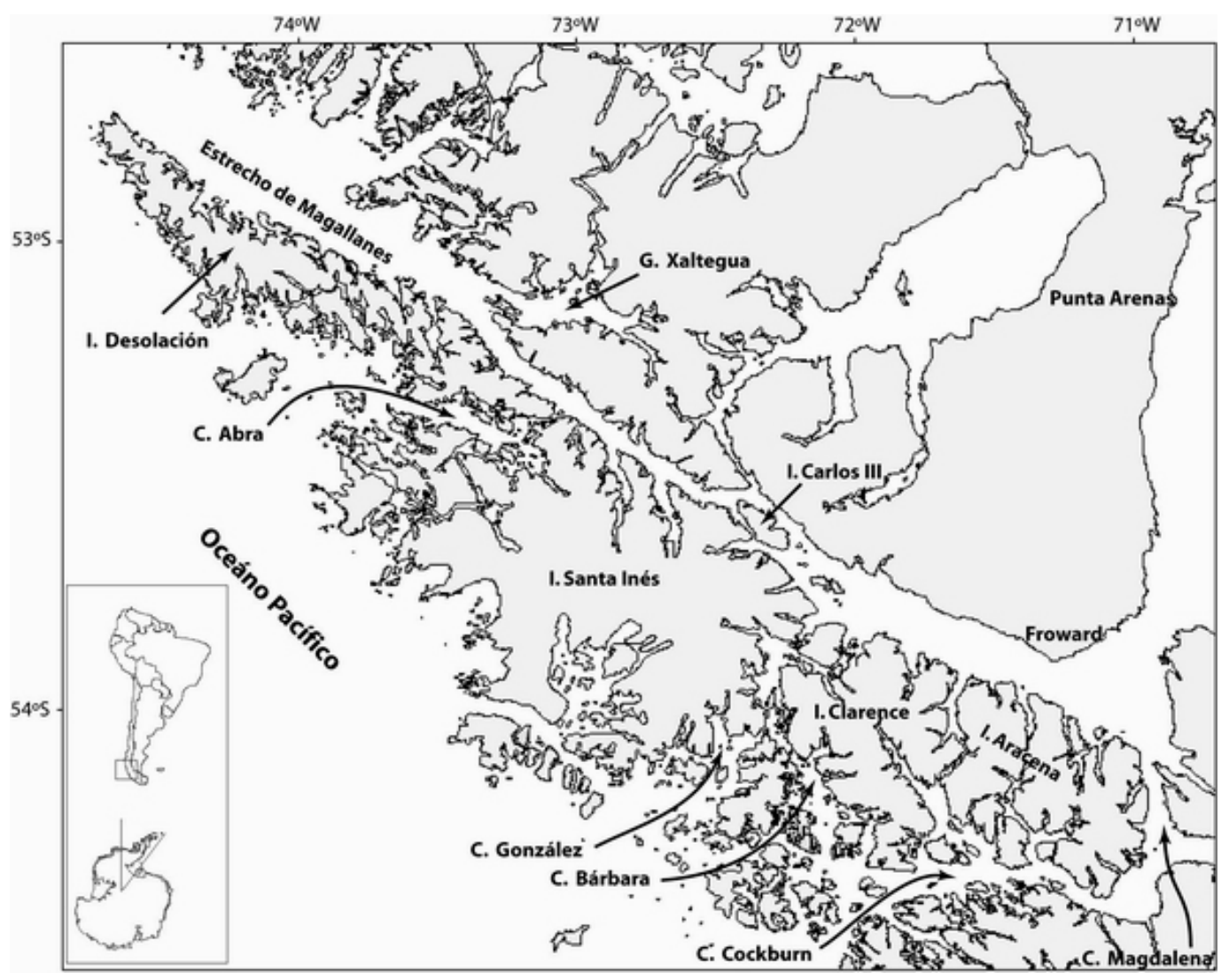

Figura 1

Área de estudio en el estrecho de Magallanes y canales adyacentes

Study area of the Magellan Strait and adjacent channels

Tabla 1

Veranos australes y períodos en que se realizaron las prospecciones en el estrecho de Magallanes y canales adyacentes

Austral summer seasons and survey periods in the Magellan Strait and adjacent channels

\begin{tabular}{ccc}
\hline $\begin{array}{c}\text { Veranos } \\
\text { australes }\end{array}$ & Período de prospecciones & $\begin{array}{c}\text { Esfuerzo } \\
\text { (No de días) }\end{array}$ \\
\hline $2002-2003$ & 20/03/2003-23/03/2003 & 4 \\
$2003-2004$ & $26 / 12 / 2003-30 / 12 / 2003$ & 5 \\
& $07 / 01 / 2004-11 / 01 / 2004$ & 5 \\
& $08 / 02 / 2004-17 / 02 / 2004$ & 10 \\
$2004-2005$ & $06 / 03 / 2004-12 / 03 / 2004$ & 7 \\
& $08 / 12 / 2004-09 / 12 / 2004$ & 2 \\
& $16 / 12 / 2004-20 / 12 / 2004$ & 5 \\
& $13 / 01 / 2005-16 / 01 / 2005$ & 4 \\
& $03 / 02 / 2005-18 / 02 / 2005$ & 16 \\
& $14 / 03 / 2005-21 / 03 / 2005$ & 8 \\
& $22 / 04 / 2005-24 / 04 / 2005$ & 3 \\
\hline
\end{tabular}

Diez prospecciones al área de estudio fueron planificadas y desarrolladas por el grupo de Biología Marina del Centro de Estudios del Cuaternario FuegoPatagonia y Antártica (CEQUA), entre marzo 2003 y abril 2005 (Tabla 1). Los cruceros se realizaron mensualmente a bordo del motovelero "Chonos", con un esfuerzo total de búsqueda y seguimiento de ballenas jorobadas de $657 \mathrm{~h}$ con buenas condiciones de navegación y observación (Tabla 2).

Durante cada avistamiento, se registraron los datos usuales conformados por: fecha, hora, ubicación y posición geográfica, nombre del lugar, dirección de los animales, número de animales, presencia de crías, comportamiento ante la embarcación (reacción, compactación del grupo, dinámica de la estructura del grupo y actividad dominante), así como las condiciones oceanográficas y de visibilidad del momento. Cada uno de los ejemplares avistados fueron clasificados subjetivamente, basados en la experiencia de los 
Tabla 2

Esfuerzo de prospección expresado en número de millas navegadas, número de días navegados y número de horas de avistamiento efectivo, por verano austral

Survey effort expressed as total nautical miles searched, days of navegation and sightings hours by season

\begin{tabular}{ccccc}
\hline Verano austral & $\begin{array}{c}\text { Esfuerzo } \\
\text { (millas navegadas) }\end{array}$ & \multicolumn{2}{c}{$\begin{array}{c}\text { Esfuerzo } \\
\text { (horas) }\end{array}$} & $\begin{array}{c}\text { Esfuerzo } \\
\text { (número de días navegados) }\end{array}$ \\
\hline $2002-2003$ & 192 & 24,11 & 11,45 & 4 \\
$2003-2004$ & 1.657 & 228,58 & 57,44 & 27 \\
$2004-2005$ & 2.048 & 255,17 & 78,01 & 38 \\
Total & 3.987 & $\mathbf{5 0 8 , 2 6}$ & $\mathbf{1 4 7 , 3 0}$ & $\mathbf{6 9}$ \\
\hline
\end{tabular}

autores, en adultos y jóvenes de acuerdo a la combinación del tamaño corporal relativo, altura relativa del soplo y envergadura de su aleta caudal; mientras que los ejemplares menores a $10 \mathrm{~m}$ acompañados por un adulto (presumiblemente la madre) fueron clasificados como crías. Además, se determinó el sexo de la mayoría de los ejemplares foto-identificados mediante métodos moleculares (Gilson et al. 1998) de la biopsia de piel obtenida.

Los ejemplares de $M$. novaeangliae se individualizaron a partir del patrón de coloración de la superficie ventral de su aleta caudal (Katona et al. 1979, Glockner \& Venus 1983) y complementariamente, en la forma y característica de su aleta dorsal. Fotografías de esas partes de la ballena fueron tomadas con una cámara fotográfica SLR Nikon F-100 con objetivos de 70-210 $\mathrm{mm} \mathrm{f} / 2.8$ y de $300 \mathrm{~mm} \mathrm{f/4.5,} \mathrm{utilizando} \mathrm{película} \mathrm{a} \mathrm{color}$ de 200 ASA. Para el análisis de la filopatría, se seleccionaron las mejores fotografías de cada individuo por crucero, almacenándose en una carpeta separada.

Con la información básica de terreno y de cada fotografía en particular, se estimó la tasa de retorno de la ballena jorobadas al área de alimentación del estrecho de Magallanes en diferentes veranos australes, basado en el número de ejemplares foto-identificados $\mathrm{y}$ recapturados en los veranos siguientes mediante la expresión:

Tasa de retorno $=\left(\mathrm{N}_{\text {rec }} / \mathrm{N}_{\text {tot }}\right) \times 100$

Donde:

$\mathrm{N}_{\text {rec }}=$ Total de ballenas foto-identificados recapturados

$\mathrm{N}_{\text {tot }}=$ Total de ballenas foto-identificadas
Para las comparaciones se utilizaron pruebas estadísticas del Chi cuadrado $\left(\mathrm{X}^{2}\right)$ usando el programa Statistical 5.1 (StatSoft 1997). Diferencias significativas fueron consideradas a un nivel de $P<0,05$.

\section{Resultados}

En los veranos australes 2002-2003, 2003-2004 y 20042005, un total de 104 ejemplares de ballena jorobada fue foto-identificado en el área de estudio, correspondiendo éstos a 67 animales diferentes. Durante el verano austral 2002-2003 se foto-identificaron 11 animales; en el verano 2003-2004 se foto-identificaron 35 animales de los cuales 27 fueron nuevos; y durante la última temporada se foto-identificaron 58 ballenas de las cuales 29 correspondieron a animales nuevos.

De las 11 ballenas individualizadas durante marzo 2003, 10 fueron recapturadas en los veranos siguientes. De éstas, ocho individuos regresaron en el verano 20032004, mientras que nueve ejemplares también regresaron en el verano subsiguiente (2004-2005), representando una tasa de retorno de 72,7\% y 81,8\%, respectivamente. Además, siete ejemplares han retornado durante dos veranos consecutivos, representando una tasa de retorno de 63,6\%. Así mismo, 20 de los 27 ejemplares nuevos individualizados durante el verano 2003-2004, retornaron al área en la temporada de verano siguiente (2004-2005) (Tabla 3)

La filopatría al área de estudio de los ejemplares individualizados durante los dos primeros veranos fue alta y similar entre ellas, obteniéndose una tasa global de $90,9 \%$ de retorno de ballena jorobada fotoidentificadas en el verano 2002-2003, y de 74,1\% de retorno de los ejemplares nuevos individualizados durante el verano 2003-2004 (Tabla 4). 
Tabla 3

Número de ejemplares de $M$. novaeangliae foto-identificados durante los veranos 2002-2003 y 2003-2004 y recapturados en los veranos siguientes en el área de estudio

Number of M. novaeangliae photo-identified during 2002-2003 and 2003-2004 summer seasons and recaptured in following seasons in the study area

\begin{tabular}{|c|c|c|c|c|}
\hline \multirow[t]{2}{*}{$\mathbf{N}^{\circ}$} & \multirow[t]{2}{*}{$\begin{array}{c}\text { Código de } \\
\text { foto-identificación }\end{array}$} & \multirow[t]{2}{*}{$\begin{array}{c}\text { Verano austral } \\
\text { foto ID }\end{array}$} & \multicolumn{2}{|c|}{$\begin{array}{l}\text { Fecha de } \\
\text { recaptura }\end{array}$} \\
\hline & & & 2003-2004 & 2004-2005 \\
\hline 1 & \#001 & 2002-2003 & -- & 19/03/05 \\
\hline 2 & $\# 002$ & 2002-2003 & -- & -- \\
\hline 3 & $\# 003$ (= \#041) & 2002-2003 & $12 / 02 / 04$ & $10 / 02 / 05$ \\
\hline 4 & $\# 004(=\# 021)$ & 2002-2003 & 28/12/03 & 09/02/05 \\
\hline 5 & $\# 005$ (= \#015) & 2002-2003 & $27 / 12 / 03$ & $16 / 12 / 04$ \\
\hline 6 & \#006 & 2002-2003 & -- & $15 / 01 / 05$ \\
\hline 7 & $\# 007$ (= \#044) & 2002-2003 & $15 / 02 / 04$ & $15 / 01 / 05$ \\
\hline 8 & \#008 (= \#012 y 017) & 2002-2003 & $27 / 12 / 03$ & -- \\
\hline 9 & $\# 009$ (= \#018) & 2002-2003 & $27 / 12 / 03$ & $16 / 12 / 04$ \\
\hline 10 & $\# 010(=\# 042)$ & 2002-2003 & $12 / 02 / 04$ & $19 / 12 / 04$ \\
\hline 11 & \#011 (= \#019 y 058) & 2002-2003 & 28/12/03 & $14 / 01 / 05$ \\
\hline 12 & $\# 013$ & 2003-2004 & -- & 08/02/05 \\
\hline 13 & \#014 & 2003-2004 & -- & $19 / 12 / 04$ \\
\hline 14 & \#016 & 2003-2004 & -- & $19 / 03 / 05$ \\
\hline 15 & \#020 & 2003-2004 & -- & 09/02/05 \\
\hline 16 & \#022 & 2003-2004 & -- & -- \\
\hline 17 & \#023 & 2003-2004 & -- & $20 / 12 / 04$ \\
\hline 18 & \#024 & 2003-2004 & -- & -- \\
\hline 19 & \#025 & 2003-2004 & -- & $04 / 02 / 05$ \\
\hline 20 & \#026 & 2003-2004 & -- & $14 / 01 / 05$ \\
\hline 21 & \#027 & 2003-2004 & -- & $13 / 01 / 05$ \\
\hline 22 & \#028 & 2003-2004 & -- & $13 / 01 / 05$ \\
\hline 23 & \#029 & 2003-2004 & -- & $22 / 04 / 05$ \\
\hline 24 & $\# 030$ & 2003-2004 & -- & -- \\
\hline 25 & \#031 & 2003-2004 & -- & -- \\
\hline 26 & \#032 & 2003-2004 & -- & $15 / 01 / 05$ \\
\hline 27 & \#033 & 2003-2004 & -- & 05/02/05 \\
\hline 28 & \#034 & 2003-2004 & -- & 09/02/05 \\
\hline 29 & \#035 & 2003-2004 & -- & $14 / 01 / 05$ \\
\hline 30 & \#036 & 2003-2004 & -- & $15 / 01 / 05$ \\
\hline 31 & \#037 & 2003-2004 & -- & $15 / 01 / 05$ \\
\hline 32 & \#038 & 2003-2004 & -- & 06/02/05 \\
\hline 33 & \#039 & 2003-2004 & -- & -- \\
\hline 34 & \#040 & 2003-2004 & -- & $14 / 01 / 05$ \\
\hline 35 & \#043 & 2003-2004 & -- & -- \\
\hline 36 & \#045 & 2003-2004 & -- & $16 / 12 / 04$ \\
\hline 37 & \#046 & 2003-2004 & -- & $19 / 03 / 05$ \\
\hline 38 & $\# 047$ & 2003-2004 & -- & -- \\
\hline
\end{tabular}




\section{Tabla 4}

\section{Tasa de retorno (\%) al estrecho de Magallanes y canales adyacentes de los ejemplares de M. Novaeangliae foto-identificados durante los veranos 2002-2003 y 2003-2004}

Rate of return (\%) to the Magellan Strait and adjacent channels of M. novaeangliae photo-identified in the austral summer 2002-2003 and 2003-2004

\begin{tabular}{|c|c|c|c|c|}
\hline \multirow[t]{3}{*}{ Verano austral } & \multicolumn{3}{|c|}{ Total ballenas únicas } & \multirow{3}{*}{$\begin{array}{l}\text { \% Retorno } \\
\text { observado }\end{array}$} \\
\hline & \multirow[t]{2}{*}{ Foto-identificadas } & \multicolumn{2}{|c|}{ Recapturadas } & \\
\hline & & Observadas & Esperadas & \\
\hline 2002-2003 & 11 & 10 & 09 & 90,9 \\
\hline 2003-2004 & 27 & 20 & 21 & 74,1 \\
\hline Total & 38 & & & $\begin{array}{c}78,9 \\
X^{2}=0,15(P=0,69)\end{array}$ \\
\hline
\end{tabular}

Por su parte, la filopatría de los ejemplares fotoidentificados en el área de estudio en diferentes veranos australes, considerando las temporadas 1 y 2 en conjunto, no mostró ser significativamente diferente entre adultos y jóvenes $\left(\mathrm{X}^{2}=0,04 ; P=0,82 ; \mathrm{n}=30\right)$. Así mismo, la filopatría por sexo tampoco mostró ser significativamente diferentes entre machos y hembras $\left(\mathrm{X}^{2}=0,33 ; P=0,56 ; \mathrm{n}=27\right.$ ), indicando que la filopatría de la ballena jorobada al área de alimentación del estrecho de Magallanes se mantiene en proporciones de 50:50.

\section{Discusión}

Los resultados presentados en este estudio indican que la filopatría hacia esta segunda área de agregación, correspondiente al estrecho de Magallanes, en diferentes veranos australes, es alta (74,1\% - 90,9\%). Esta alta tasa de retorno al área del estrecho de Magallanes y canales adyacentes en los veranos australes es similar a la informada para otras áreas de alimentación. Así por ejemplo, para Alaska se ha informado una filopatría del 70\% de los ejemplares de ballena jorobada (Gabriele $1997^{3}$ ) y de $71 \%$ - $88 \%$ para el área de alimentación de California (Calambokidis et al. 1996). Varios autores también han informado sobre

3 Gabriele CM. 1997. Comparison of humpback whale group dynamics between Hawaii and Alaska. In: Report of the Workshop to Assess Research and Other Needs and Opportunities Related to Humpback Whale Management in the Hawaiian Islands. Compiled by P.M. Payne, B. Phillips \& E. Nitta. U.S. Department of Commerce, NOAA Technical Memo. NMFS-OPR-11. 31-37 pp. una alta filopatría hacia otras áreas de alimentación en el Pacífico norte (Calambokidis et al. 1997², Urbán et al. 2000), así como en el Atlántico norte (Clapham et al. 1993).

Otros resultados de estudios de foto-identificación han documentado que los individuos de $M$. novaeangliae de las poblaciones del Pacífico norte y de la población del Atlántico norte tienden a retornar a la mismas áreas de alimentación y reproducción en años subsecuentes (Darling \& McSweeney 1985, Baker et al. 1986, von Ziegesar et al. 1994, Calambokidis et al. 1996), aunque algunos estudios sugieren que la filopatría de los ejemplares de esta especie sería mayor hacia las áreas de alimentación que hacia las áreas de reproducción. Un ejemplo de lo anterior, es el caso de la población mexicana de ballena jorobada que exhibe una alta filopatría hacia áreas específicas de alimentación y consistentes retornos migratorios a la región de reproducción en invierno (Urbán et al. 2000).

No obstante de esta tendencia general, movimientos de animales han sido registrados entre distintas áreas de alimentación y entre distintas áreas de reproducción en el Pacífico norte (Darling \& Jurasz 1983, Darling \& McSweeney 1985, Baker et al. 1986, Perry et al. 1990, Straley 1990, Darling \& Cerchio 1993, von Ziegesar et al. 1994, Calambokidis et al. 1996, 1997, Sladen et al. 1999, Waite et al. 1999). Además, algunos autores han planteado la hipótesis de que una fracción de individuos, probablemente hembras, permanecerían en las áreas de alimentación durante todo el año (Chittleborough 1958, Craig \& Herman 1997, Brown et 
al. 1995), aunque dicha hipótesis aún no ha sido comprobada.

Algunas evidencias apuntan a indicar que la filopatría de $M$. novaeangliae hacia las zonas de alimentación es transmitida maternalmente a su cría, como un resultado de la experiencia adquirida durante el primer año de vida (Baker et al. 1990, 1994, PalsbØll et al. 1995). Más aún, las notorias segregaciones de haplotipos mitocondriales entre poblaciones de esta especie han sido interpretadas como una consecuencia de la filopatría transmitida maternalmente a la cría hacia los distintos destinos migratorios, los cuales se han mantenido sobre un tiempo de escala evolutivo (PalsbØll et al. 1995, Larsen et al. 1996). En este sentido, una cría (ejemplar \#009) foto-identificada en marzo de 2003 junto a su madre en las cercanías de Isla Carlos III, estrecho de Magallanes, a sido re-avistada como un joven independiente durante los dos veranos consecutivos (2003-2004 y 2004-2005), en las aguas adyacentes a dicha isla (ver Tabla 3).

Los estudios sobre la filopatría por sexos han sido realizados sólo en las áreas de reproducción, sugiriendo algunos autores que los machos muestran una mayor filopatría que las hembras (Craig \& Herman 1997), mientras que otros autores sugieren lo contrario: que los machos serían más móviles que las hembras en las áreas invernales (Garrigue et al. 2000), aunque Baker et al. (1994) indican que la posible diferencia sexual en la filopatría no sería tan obvia, y que la ballena jorobada usaría una combinación de estrategias para retornar a su destino migratorio, como la impronta y la característica genética. No obstante, la filopatría a las áreas de alimentación por sexo y categorías de clase/edad no ha sido evaluada.

En este estudio, el número de ejemplares machos vs hembras así como la cantidad de adultos vs jóvenes, se mantuvo en proporciones similares, respectivamente. Esta similitud en la proporción sexual también fue observada por Olavarría (1999) en el área de alimentación de la costa occidental de la península Antártica, indicando que la proporción de sexos de 48 ejemplares muestreados en diferentes años no fue estadísticamente diferente a la razón 50:50.

Otras evidencias que apuntarían sobre la proporcionalidad igualitaria de la fidelidad sexual hacia las áreas de alimentación, puede ser obtenido de algunos datos de caza de esta especie. Tomilin (1967) indica que, de las ballenas cazadas en la Antártica entre los años 1933 y 1938, el 55,7\% fueron hembras, aunque propone que la diferencia de hembras vs machos puede ser explicada por el afán de los arponeros a cazar animales más grandes. Sin embargo, datos de caza de la CBI en el Área V para 16 años post guerra (1947 a 1963) indican que los ejemplares de ballena jorobada cazados fueron principalmente machos (60\%). Una relación similar fue observada en los datos de caza de los balleneros rusos Slava y Sovietskaya Ukraina donde se habrían cazado un 54,23\% de machos y un 45,77\% de hembras (Mikhalev 20004).

Finalmente, la alta tasa de retorno de los ejemplares foto-identificados en los diferentes veranos australes obtenida en este trabajo para el área del estrecho de Magallanes, indica que ésta constituye otra zona de alimentación para al menos una parte de la población de ballena jorobada del Pacífico suroriental.

\section{Agradecimientos}

Se agradece al colega Carlos Olavarría B. y al Laboratorio de Biología Molecular del Dr. C.S. Baker, Universidad de Auckland, por la determinación genética de los sexos de los ejemplares muestreados de ballena jorobada. Además, se agradece a la tripulación del motovelero "Chonos", en nombre de su armador Francisco Ayarza O. y a todos los colegas que han participado en las diferentes campañas de terreno. Finalmente, se agradece al Gobierno Regional de Magallanes, CONICYT y a la Dirección del Centro de Estudios del Cuaternario (CEQUA) y del Instituto Antártico Chileno (INACH) por las facilidades para el estudio de esta especie en el estrecho de Magallanes y a los colegas P. Acuña y C. Olavarría por sus comentarios ofrecidos a una versión previa de este trabajo.

\section{Literatura citada}

Baker CS, LM Herman, A Perry, WS Lawton, JM Straley, AA Wolman, GD Kaufman, HE Winn, JD Hall, JM Reinke \& J Östman. 1986. Migratory movement and population structure of humpback whale (Megaptera novaeangliae) in the central and eastern North Pacific. Marine Ecology Progress Series 31: 105-119.

\footnotetext{
${ }^{4}$ Mikhalev Y. 2000. Biological characteristics of humpbacks taken in Antarctic Area V by the whaling fleets Slava and Sovietskaya Ukraina. Document SC/52/IAWP7 for the Scientific Committee of the International Whaling Commission. Adelaide, Australia. 18 pp.
} 
Baker CS, SR Palumbi, RH Lambertsen, MT Weinrich, J Calambokidis \& SJ O'Brien. 1990. Influence of seasonal migration on geographic distribution of mitochondrial DNA haplotypes in humpback whales. Nature 344: 238-340.

Baker CS, RW Slade, JL Bannister, RB Abernethy, MT Weinrich, J Lien, J Urbán, PJ Corkeron, J Calambokidis, O Vasquez \& SR Palumbi. 1994. Hierarchical structure of mitochondrial DNA gene flow among humpback whales Megaptera novaeangliae, world-wide. Molecular Ecology 3: 313-327.

Brown MR, PJCorkeron, PT Hale, KW Schultz \& MM Bryden. 1995. Evidence for a sex-segregated migration in the humpback whale (Megaptera novaeangliae). Proceeding of the Royal Society of London, Serie B, Biological Science 259: 229-234.

Calambokidis J, GH Steiger, JR Evenson, KR Flynn, KC Balcomb, DE Claridge, P Bloedel, JM Straley, CS Baker, O von Ziegesar, ME Dahlheim, JM Waite, JD Darling, G Ellis \& GA Green. 1996. Interchange and isolation of humpback whales off California and other North Pacific feeding grounds. Marine Mammal Science 12: 215-226.

Calambokidis J, GH Steiger, K Rasmussen, J Urbán, KC Balcomb, $P$ Ladrón de Guevara, M Salinas, JK Jacobsen, CS Baker, LM Herman, S Cerchio \& JD Darling. 2000. Migratory destinations of humpback whales that feed off California, Oregon and Washington. Marine Ecology Progress Series 192: 295-304.

Calambokidis J, GH Steiger, JM Straley, LM Herman, S Cerchio, DR Salden, J Urbán, JK Jacobson, O vonZiegesar, KC Balcomb, CM Gabrielle, ME Dahlheim, S Uchida, G Ellis, Y Miyamura, P Ladrón de Guevara, M Yamaguchi, F Sato, SA Mizroch, L Schlender, K Rasmussen, J Barlow \& TJ Quinn II. 2001. Movements and population structure of humpback whales in the North Pacific. Marine Mammal Science 17: 769-794.

Chaloupka M \& MB Osmond. 1999. Spatial and seasonal distribution of humpback whales in the Great Barrier Reef Region. En: Musick JA (ed). Life in the slow lane: Ecology and conservation of long-lived marine animals, pp. 89-106. American Fisheries Society Symposium 23.

Chittleborough RG. 1958. The breeding cycle of the female humpback whale, Megaptera nodosa (Bonneterre). Australian Journal of Marine and Freshwater Research 6: 3-29.

Clapham PJ. 2000. The humpback whale: seasonal feeding and breeding in a baleen whale. En: Mann J, RC Connor, PL Tyack \& H Whitehead (eds). Cetacean Societies: field studies of whales and dolphins, pp. 173-196. Chicago, University of Chicago Press.
Clapham PJ, LS Baraff, CA Carlson, MA Christian, DK Mattila, CA Mayo, MA Murphy \& S Pittman. 1993. Seasonal occurrence and annual return of humpback whales in the southern Gulf of Maine. Canadian Journal of Zoology 71: 440-443.

Craig AS \& LM Herman. 1997. Sex differences in site fidelity and migration of humpback whales (Megaptera novaeangliae) to the Hawaiian Islands. Canadian Journal of Zoology 75: 1923-1933.

Darling JD \& CM Jurasz. 1983. Migratory destination of North Pacific humpback whales (Megaptera novaeangliae). En: Payne R (ed). Communication and behavior of whales, pp. 359-368. Westview Press, Boulder, Colorado.

Darling JD \& DJ McSweeney. 1985. Observations on the migrations of North Pacific humpback whales (Megaptera novaeangliae). Canadian Journal of Zoology 63: 308-314.

Darling JD \& S Cerchio. 1993. Movement of a humpback whale (Megaptera novaeangliae) between Japan and Hawaii. Marine Mammal Science 9 (1): 84-89.

Garrigue C, PH Forestell, J Greaves, P Gill, P Naessig, NM Patenaude \& CS Baker. 2000. Migratory movements of humbpack whales (Megaptera noaveangliae) between New Caledonia, East Australia and New Zealand. Journal of Cetacean Research and Management 2: 111-115.

Garrigue C, A Aguayo, VLU Amante-Helweg, CS Baker, S Caballero, $P$ Clapham, $R$ Constantine, J Denkinger, $M$ Donoghue, L Flórez-González, J Greaves, N Hauser, C Olavarría, C Pairoa, H Peckham \& M Poole. 2002. Movements of humpback whales in Oceania, South Pacific. Journal of Cetacean Reserach and Management 4: 255-260.

Gibbons J, F Gazitúa \& C Venegas. 2000. Cetáceos en el estrecho de Magallanes y Senos Otway, Skyring y Almirantazgo. Anales del Instituto de la Patagonia, Serie Ciencias Naturales (Chile) 28: 107-118.

Gibbons J, JC Capella \& C Valladares. 2003. Rediscovery of a humpback whale (Megaptera novaeangliae) feeding ground in the Straits of Magellan, Chile. Journal of Cetacean Research and Management 5 (2): 203-208.

Gilson A, M. Syvanen, K Levine \& J Banks. 1998. Deer gender determination by polymerase chain reaction: validation study and application to tissues, bloodstain and hair forensic samples from California. California Fish and Game 84: 159-169.

Glockner I \& R Venus. 1983. Identification, growth rate and behaviour of humpback whale (Megaptera novaeangliae) cows and calves in the waters off Maui, Hawai, 1977-79. En: Payne R (ed). Communication and behaviour of whales, pp. 223-258. Westview Press, Boulder, Colorado. 
Katona S, B Baxter, O Brazier, S Kraus, J Perkins \& H Whitehead. 1979. Identification of humpback whales by fluke photographs. En: Winn HE \& BL Olla (eds). Behavior of Marine Animals 4: 33-34. Plenum Press, New York

Larsen AH, J Sigurjónsson, N Øien, G Vikingsson \& $\mathbf{P}$ PalsbØll. 1996. Population genetic analysis of nuclear and mitochondrial loci in skin biopsies collected from central and northeastern North Atlantic humpback whales (Megaptera novaeangliae): population identity and migratory destinations. Proceeding of The Royal Society of London, Serie B Biological Science 263: 1611-1618.

Mackintosh NA. 1965. The stocks of whales. 232 pp. Fishing News (Books) Ltd. London.

Mikhalev Y. 1997. Humpback whales, Megaptera novaeangliae in the Arabian Sea. Marine Ecology Progress Series 149: 13-21.

Olavarría C. 1999. Identidad genética de la ballena jorobada (Megaptera novaeangliae, Borowski 1781) en las aguas adyacentes a la Península Antártica. Tesis de Biología Marina. Facultad de Ciencias del Mar, Universidad de Valparaíso, 62 pp.

Omura H. 1953. Biological study on humpback whales in the Antarctic whaling areas IV and V. Scientific Report of Whales Research Institute 8: 81-102.

Palsbøll PJ, PJ Clapham, DK Mattila, F Larsen, R Sears, HR Siegismund, J Sigurjónsson, $O$ Vásquez \& $P$ Arctander. 1995. Distribution of Mt DNA haplotypes in North Atlantic humpback whales: the influence of behavior on population structure. Marine Ecology Progress Series 116: 1-10.

Perry A, CS Baker \& LM Herman. 1990. Population characteristics of individually identified humpback whales in the central and eastern North Pacific: A summary and critique. Report of the International Whaling Commission, Special Issue 12: 307-317.

Rice DW. 1998. Marine mammals of the world. Systematics and Distribution. Special Publication 4, 231 pp. The Society for Marine Mammalogy, Allen Press Inc., Lawrence, Kansas.

Simmons ML \& HE Marsh. 1985. Sightings of humpback whales in Great Barrier Reef waters. Scientific Reports of the Whales Research Institute 37: 31-46.
Sladen DR, LM Herman, M Yamaguchi \& F Sato. 1999. Multiple visits of individual humpback whales (Megaptera novaeangliae) between the Hawaiian and Japanese winter grounds. Canadian Journal of Zoology 77: 504-508.

Soler M, J Carranza, A Cordero, J Moreno, JC Senar \& JJ Soler. 2001. Traducción al español de los términos ingleses más conflictivos utilizados en Etología, Ecología y Evolución. Etología 9: 43-46.

StatSoft. 1997. STATISTICA for Windows (Computer program manual). Tulsa, OK. Oklahoma.

Stevick PT, A Aguayo, J Allen, IC Avila, J Capella, C Castro, K Chater, M Engel, F Felix, L FlórezGonzález, A Freitas, B Hasse, M Llano, L Lodi, E Munoz, C Olavarría, E Secchi, M Scheidat y S Siciliano. 2004. A note on the migrations of individually identified humpback whales between the Antarctic Peninsula and South America. Journal of Cetacean Research and Management 6(2): 109-113.

Straley JM. 1990. Fall and winter ocurrence of humpback whales (Megaptera novaeangliae) in Southeastern Alaska. Report of the International Whaling Commission Special Issue 12: 319-323.

Tomilin AG. 1967. Mammals of the USSR and adjacent countries. Vol. IX. Cetacea, 717 pp. Israel Program for Scientific Translations, Jerusalem.

Urbán J, A Jaramillo, A Aguayo, P Ladrón de Guevara, M Salinas, C Álvarez, L Medrano, JK Jacobsen, KC Balcomb, DE Claridge, J Calambokidis, GH Steiger, JM Straley, O Von Ziegesar, JM Waite, S Mizroch, ME Dahlhem, JD Darling \& CS Baker. 2000. Migratory destinations of humpback whales wintering in the Mexican Pacific. Journal of Cetacean Research and Management 2 (2): 101-110.

Von Ziegesar O, E Miller \& ME Dahlheim. 1994. Impacts on humpback whales in Prince Williams Sound. En: Loughlin TR (ed), Marine Mammals and the Exxon Valdez, pp. 173-191. Academic Press, San Diego.

Waite JM, ME Dahlheim, RC Hobbs \& SA Mizroch. 1999. Evidence of a feeding aggregation of humpback whales (Megaptera novaeangliae) around Kodiak Island, Alaska. Marine Mammal Science 15 (1): 210-220. 